\title{
Cestas orgânicas: um estudo de caso de cadeia curta agroalimentar familiar
}

\author{
Organic baskets: a case study of family agri-food short chain \\ canastas orgánicas: um estudio de caso de cadena corta agroalimentaria familiar
}

Recebido: 19/08/2021 | Revisado: 25/08/2021 | Aceito: 31/10/2021 | Publicado: 01/11/2021

\author{
Aguinaldo Eduardo de Souza \\ ORCID: https://orcid.org/0000-0001-6100-752X \\ Universidade Paulista, Brasil \\ Faculdade São Vicente, Brasil \\ E-mail: souza.eduaguinaldo@gmail.com \\ João Gilberto Mendes dos Reis \\ ORCID: https://orcid.org/0000-0001-6409-2299 \\ Universidade Paulista, Brasil \\ E-mail: joao.reis@docente.unip.br \\ Luciana Melo Costa \\ ORCID: https://orcid.org/0000-0002-2275-743X \\ Universidade Federal de São Paulo, Brasil \\ E-mail: lueducita@yahoo.com.br \\ Oduvaldo Vendrametto \\ ORCID: https://orcid.org/0000-0003-2430-6138 \\ Universidade Paulista, Brasil \\ E-mail: oduvaldove@gmail.com
}

\begin{abstract}
Resumo
A demanda por alimentos mais saudáveis, produzidos localmente, com características da cultura local, garantindo justiça social, popularizou as cadeias alimentares curtas. Essas redes têm se mostrado substituto confiável para as cadeias de suprimentos convencionais. Além disso, possibilita o empoderamento socioeconômico do agricultor familiar, garantindo receitas mais estáveis. O presente estudo teve como objetivo analisar implantação de uma cadeia curta através de uma rede de distribuição local de cestas orgânicas, em uma propriedade familiar. Os resultados mostraram um crescimento de mais de $400 \%$ na produção e uma valorização de $1.500 \%$ no preço de vendas das cestas no período 2016/2019, a partir do desenvolvimento e consolidação da cadeia curta, aqui denominada "Cadeia Curta Familiar Agroecológica".
\end{abstract}

Palavras-chave: Agricultura familiar; Cadeia curta familiar agroecológica; Cestas orgânicas.

\begin{abstract}
The demand for healthier foods, produced locally, with characteristics of local culture, which guarantee social justice, has popularized the Short Food Chains. These networks have proven to be a reliable substitute for conventional supply chains. In addition, it enables the socioeconomic empowerment of the family farmer, ensuring more stable revenues. The present study aimed to analyze the development of a short chain through a local distribution network of organic baskets, a family farm property. The results showed a growth of more than $400 \%$ in production and an increase of $1,500 \%$ in the sales price of the baskets in the period 2016/2019, from the development and consolidation of the short chain, here called "Agroecological Family Short Chain".
\end{abstract}

Keywords: Family farming; Family agri-food short chain; Organic baskets.

\section{Resumen}

La demanda de alimentos más saludables, producidos localmente, con características de la cultura local, garantizando la justicia social, popularizó las cadenas alimentarias cortas. Estas redes han demostrado ser un sustituto confiable de las cadenas de suministro convencionales. Además, posibilita el empoderamiento socioeconómico del agricultor familiar, asegurando ingresos más estables. Este estudio tuvo como objetivo analizar la implementación de una cadena corta a través de una red de distribución local de canastas orgánicas, en una propiedad familiar. Los resultados arrojaron un crecimiento de más del $400 \%$ en la producción y un incremento del $1.500 \%$ en el precio de venta de las canastas en el período 2016/2019, a partir del desarrollo y consolidación de la cadena corta, aquí denominada "Cadena Corta Agroecológica Familiar".

Palabras clave: Agricultura familiar; Cadena corta agroalimentaria familiar; Cestas orgánicas. 


\section{Introdução}

Nos últimos anos tem se observado no mundo um crescente movimento ligado ao consumo de alimentos produzidos localmente. O Local Food, significa que a comida foi cultivada nas proximidades físicas do consumidor final (Sacchi et al. 2018). Esse movimento teve seu início e consolidação nos Estados Unidos e Europa, e atualmente tem se disseminado por outras regiões do globo, inclusive no Brasil (Coelho et al., 2018).

As novas formas de produção e consumo ganharam destaque a partir de 1990 com o chamado movimento "quality turn", marcado por consumidores mais exigentes e conscientes que observam valores de confiança, integração e localidade como critérios importantes de qualidade que transcendem aspectos puramente tangíveis (Goodman, 2002; Goodman, 2003).

Desse modo, a busca por alimentos sustentáveis culminou no desenvolvimento das Redes Alimentares Alternativas (Alternative Food Networks - AFN) através do sistema alimentar local (Local Food) se consolidou como uma alternativa viável ao sistema agroalimentar tradicional Martinez et. al (2010), desempenhando um papel fundamental na qualidade alimentar (Brunori, 2003).

Todorovic et al. (2018) asseveram que os modelos de redes alimentares tradicionais (distribuição em larga escala, operadas pelas cadeias globais de suprimentos), podem apresentar desperdício de alimentos, problemas de segurança alimentar, danos ambientais, distribuição injusta de valor agregado e lucros entre aos membros da cadeia. Além disso, a restrição de acesso a novos mercados e obtenção de preços justos pela produção, tem inviabilizado o empoderamento socioeconômico do agricultor familiar em virtude da pressão dos grandes players operadores do modelo tradicional, em busca de novos patamares de rentabilidade (Darolt et al., 2016).

Renting et al. (2003), Galli \& Brunori (2013), Duarte \& Tomé (2016), Matte et al. (2016), Anjo \& Caldas (2017), Gaucher-Petitdemange (2018) configuram cadeias curtas como redes alimentares que promovem maior aproximação produtor/consumidor, reduzindo ou eliminando o agente "intermediário". Os estudos apontam benefícios como alimentos sustentáveis atestados por sua origem, com características da cultura local e forte apelo socioeconômico, garantindo justiça social as comunidades locais.

Ademais, as cadeias curtas têm como propósito o desenvolvimento de inciativas locais, emprego de insumo de propriedades locais, baixa escala na produção com foco na qualidade em detrimento da alta produtividade (Hinrichs, 2003; Pivoto et al. 2016; Vitterso et. al 2019).

Embora haja uma percepção positiva a respeito dos benefícios em relação consumo do alimento local, o estudo busca responder questões mais amplas, tais como: 1) Redes alimentares baseadas em "Local Food" podem ser considerados modelos de Cadeia Curta Agroalimentar? 2) Geram benefícios socioeconômicos para o pequeno agricultor vinculado a agricultura familiar?

Para responder essas indagações foi realizado uma pesquisa de campo, através de um estudo de caso junto a um estabelecimento rural de agricultura familiar no Brasil. Além dessa seção introdutória, o artigo está organizado como segue: A seção 2 descreve a metodologia de pesquisa aplicada. É apresentado uma abordagem teórica de Agricultura Familiar no Brasil, Local Food e Cadeias Curtas Agroalimentares na seção 3. Em seguida é descrito os principais resultados da pesquisa de campo na seção 4. Na seção 5 é discutido os resultados do estudo de caso. E por fim, constam as conclusões acerca da pesquisa e as referências.

\section{Metodologia}

Além da revisão de literatura em busca do estado da arte de Cadeias Agroalimentares Curtas de Abastecimento, Local Food e Agricultura Familiar, o presente estudo consistiu em analisar a produção, tipos de produtos, comercialização e perfiz de 
clientes acerca das cestas de produtos orgânicos comercializados entre os anos 2016 e 2019. A metodologia aplicada pode ser caracterizada como multimétodo Cauchick-Miguel et. al (2018), ou seja, foi adotado mais de uma abordagem metodológica de pesquisa (estudo de caso, pesquisa de campo e survey). A metodologia em detalhes consistiu das seguintes etapas:

Etapa 1: Revisão literária. Foi realizado um levantamento de dados nas principais bases de consultas de trabalhos científicos (ScienceDirect, Scopus, IEEE, Scielo, Google Acadêmico, Portal de Periódicos da CAPES). Pesquisa bibliográfica é um método que visa analisar estudos sobre diversos posicionamentos acerca de trabalhos já desenvolvidos (Gil, 2008).

Etapa 2: Coleta e Análise de dados. Optou-se por uma pesquisa exploratória, de caráter descritivo. A pesquisa descritiva, segundo Cervo (2009) busca descobrir de forma precisa, a frequência com que um fenômeno ocorre e sua relação com outros. O estudo foi desenvolvido no mês de fevereiro de 2020, no Assentamento Rural na cidade Promissão (SP) através de uma pesquisa de campo junto a uma família de agricultores familiares de produção orgânica. Para tal, aplicou-se o método de pesquisa tipo survey com objetivo de avaliar as cadeias produtivas de agricultores familiares, a fim de extrair resultados acerca das práticas operacionais da logística de escoamento da produção. O survey objetiva avaliar uma amostra significativa de um problema a ser investigado a fim de extrair resultados acerca dessa amostra (Cauchick-Miguel, 2018).

Etapa 3: Discussão dos resultados. Buscou apresentar os resultados obtidos que pudessem qualificar o projeto.

\subsection{Estudo de Caso}

O estudo foi realizado na Agrovila Campinas, junto à "Família Silva Orgânicos", Assentamento Rural Reunidas na cidade de Promissão - SP (Figura 1). O Assentamento Reunidas é composto por 7 agrovilas, com mais de 630 famílias assentadas em cerca de 18 hectares cada uma.

Figura 1 - Localização propriedade "Família Silva Orgânicos".

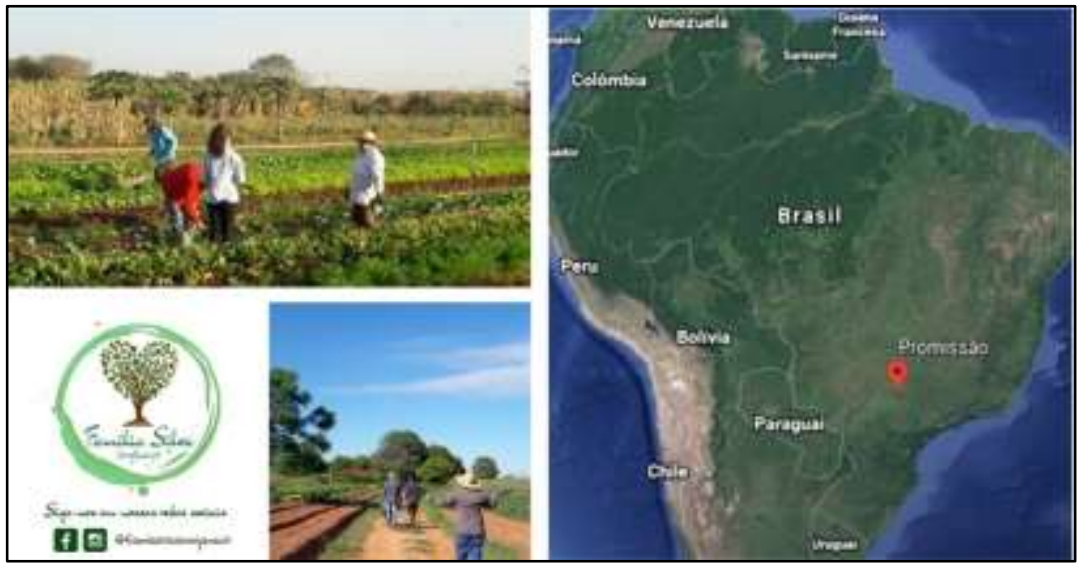

Fonte: Google Maps (2020), Autores (2020).

A monocultura da soja é o principal cultivo da família, produzida em "parceria" com outras famílias da agrovila, ocupando aproximadamente 15 hectares da propriedade. $O$ restante $(3 \mathrm{ha})$ é dedicado ao plantio legumes e hortaliças.

A força de trabalho da propriedade é majoritariamente familiar (casal e 3 filhos), salvo em casos específicos onde é contratado mão de obra esporádica no próprio assentamento, para atender alguma demanda extraordinária.

\section{Resultados}

A partir de 2016 a propriedade optou pelo cultivo de orgânicos nos $30.000 \mathrm{~m}^{2}$ dedicados a horta convencional. Definindo como estratégia comercial a venda de assinatura de cestas orgânicas. 
Antes da "virada" para o cultivo orgânico eram produzidos quiabo, abóbora, pepino e pimentas, todos comercializados no município Promissão através do mercado institucional Programa de Aquisição de Alimentos nas modalidades "Compra com Doação Simultânea 6.500 Reais/ano por agricultor" e "Compra com Doação Simultânea 8.000 Reais/ano por agricultor", ou seja, cerca de 1.200 Reais por mês. O excedente era vendido a intermediários, sujeitos a preços de mercado.

O modelo vigente de cadeias alimentares expõe a fragilidade dos agricultores familiares acerca da comercialização de seus produtos. Em que pese o mercado institucional de compra (PNAE/PAA) garantir o mínimo para escoamento da produção agrícola familiar, a dependência por "atravessadores”, ainda que seja uma alternativa viável e recorrente para muitos produtores, demonstra a vulnerabilidade dos agricultores no que tange a acesso a novos canais de distribuição, permitindo assim um desenvolvimento econômico sustentável (Darolt et al., 2016; Aguiar et al., 2018).

$\mathrm{Na}$ medida que agricultores locais inserem suas cadeias produtivas em redes alternativas visando conectar-se a novas demandas de consumo, o que se observa é um deslocamento natural da dependência de políticas públicas, bem como da figura do intermediário. Esse movimento transformacional pode ser constatado no presente estudo de caso, exemplo prático de acesso a novos mercados constituído por um sistema alimentar local, aqui denominada "Cadeia Curta Familiar Agroecológica", através da comercialização por meio de assinaturas de cestas de produtos orgânicos (Tabela 1).

Tabela 1 - Vendas de cestas orgânicas (2016-2019).

\begin{tabular}{|c|c|c|c|c|c|c|c|}
\hline Modalidade & Venda Indireta & \multicolumn{6}{|c|}{ Venda Direta } \\
\hline Ano & 2016 & \multicolumn{2}{|c|}{2017} & \multicolumn{2}{|c|}{2018} & \multicolumn{2}{|c|}{2019} \\
\hline Cidade & SRJP & Marília & SRJP & Marília & SRJP & Marília & SRJP \\
\hline Cestas/mês & 30 & 46 & 100 & 50 & 100 & 50 & 100 \\
\hline Cestas/ano & 360 & \multicolumn{2}{|c|}{1.752} & \multicolumn{2}{|c|}{1.800} & \multicolumn{2}{|c|}{1.800} \\
\hline Cesta R\$ & 15,00 & \multicolumn{2}{|c|}{30,00} & \multicolumn{2}{|c|}{40,00} & \multicolumn{2}{|c|}{50,00} \\
\hline Total R\$ & $5.400,00$ & \multicolumn{2}{|c|}{$52.560,00$} & \multicolumn{2}{|c|}{$72.000,00$} & \multicolumn{2}{|c|}{$90.000,00$} \\
\hline
\end{tabular}

Fonte: Autores (2020).

Conforme a Tabela 1, inicialmente os produtos eram comercializados em cestas compostas de 9 itens através de um empório local, onde o estabelecimento comprava e revendia quinzenalmente cerca de 15 cestas.

No entanto, o modelo de distribuição baseado em "intermediários", estava muito aquém das expectativas da propriedade em desenvolver uma rede que pudesse gerar maior valor à produção. Desse modo, após ações de difusão do projeto "cestas orgânicas", foi criado uma rede com características de econômica solidária através de um grupo de pessoas capitaneada pelo Centro de Apoio à Reforma Agrária - NARA na cidade de São José do Rio Preto - SJRP, juntamente com a família produtora.

Embora os primeiros kits de produtos entregues diretamente ao consumidor final tenham sido registrados no final de 2016 na cidade de SJRP, foi a partir de 2017 que o projeto começou a ser estruturado na forma de uma rede. Ainda em 2016, o modelo de assinatura de cestas orgânicas foi apresentado a um grupo de pessoas da cidade de Marília, com interesses relacionados a reivindicação e valorização da terra.

A rede se consolidou em 2017 com a adesão de cerca 73 famílias, 23 em Marília e 50 São J. R. Preto. Em 2018 mais duas assinaturas foram incorporadas para a cidade de Marília, perfazendo 75 assinaturas.

A cestas são montadas de acordo com a disponibilidade de produção da propriedade considerando a sazonalidade de plantio. Quinzenalmente, entre terça e quarta-feira é divulgado uma lista no grupo de Whatsapp®, principal fonte de comunicação 
da rede, dos produtos disponíveis para compor as cestas. Os assinantes confirmam os itens desejados até quinta-feira à tarde. $\mathrm{Na}$ sexta-feira a propriedade realiza a colheita e seleção dos produtos para a composição das cestas que são entregues no sábado.

A negociação é realizada na seguinte forma, os assinantes pagam na primeira entrega do mês duas ou três cestas de acordo com a quantidade de sábados mês. Para efeito de comparação de vendas (2016-2019) (Tabela 1), foi considerado os meses com quatro sábados cada um.

\section{Discussão}

A mudança na gestão das cultivares do sistema tradicional para o orgânico teve como objetivo atender à crescente demanda por alimentos de produção sustentável com garantias de qualidade nutricional Marsden et al. (2000) visando gerar valor e preços mais justos Hinrichs (2003) para a produção da propriedade (Figura 2).

Figura 2 - Evolução preços venda das cestas (2016-2019).

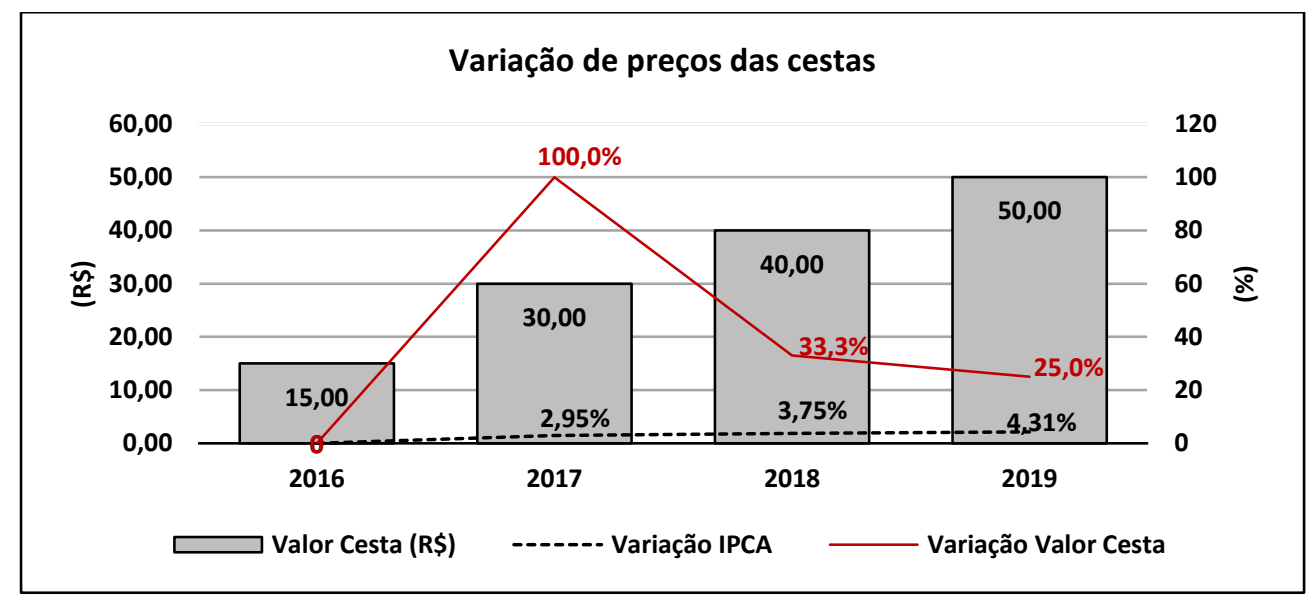

Fonte: Autores (2020).

Com a estratégia de mudança de distribuição da propriedade optando pela venda direta ao consumidor final, através da consolidação de um modelo de um modelo de cadeia curta por assinaturas no período de 2017 a 2019, conforme ilustrado na Figura 2, houve uma valorização significativa no valor das cestas de $100 \%, 33 \%$ e $25 \%$ respectivamente.

Quando comparado ao Índice de Preços ao Consumidor - IPCA IBGE (2020), principal índice utilizado para a verificação da inflação, que indicou 2,95\%, 3,75\%e 4,31\% respectivamente, os ganhos foram extremamente satisfatórios.

\section{Considerações Finais}

O estudo buscou responder as questões iniciais do objeto da pesquisa:

Primeiro: a reconfiguração produtiva da propriedade em questão, permitiu o desenvolvimento natural de uma rede alimentar local, promovendo a interação direta produtor/consumidor e a redução da cadeia logística entre o campo e a mesa, e, portanto, a construção de um modelo de Cadeias Agroalimentares Curtas.

Segundo: a propriedade apresentou um incremento de $400 \%$ na produção de cestas no período 2016/2019. O lucro bruto das vendas de assinaturas de cestas aumentou cerca de $1.500 \%$, passando de 5.400 para 90.000 reais no mesmo período.

Portanto, o que pode ser concluído é que sistemas alimentares locais, operacionalizados por meio de modelos de cadeias curtas, podem ser importantes instrumentos de empoderamento socioeconômico para o produtor familiar e a comunidade local onde ela está inserida. 
No entanto, as limitações dessa pesquisa residem no fato não mensurar a contribuição socioeconômica gerada pelo desenvolvimento e consolidação da Cadeia Curta Familiar Agroecológica na comunidade local da cidade de Promissão.

\section{Agradecimentos}

O presente trabalho foi realizado com apoio da Coordenação de Aperfeiçoamento de Pessoal de Nível Superior - Brasil (CAPES) - Código de Financiamento 001.

Família Silva Orgânicos

\section{Referências}

Aguiar, L. da C., DelGrossi, M. E., \& Thomé1, K. M. (2018). Short food supply chain: Characteristics of a family farm. Ciência Rural, 48(5). https://doi.org/10.1590/0103-8478cr20170775

Anjos, F. S. dos, \& Caldas, N. V. (2017). A dinâmica dos canais curtos de comercialização: O caso do Projeto Campagna Amica na Itália. Sociedade e Estado, 32(3), 771-792. https://doi.org/10.1590/s0102-69922017.32030010

BRASIL, G. F.-. (2003). Lei no 10.696 [Casa Civil]. Palácio do Planalto. http://www.planalto.gov.br/ccivil_03/leis/2003/L10.696.htm

BRASIL, G. F.-. (2009). Lei n 11.947 [Casa Civil]. Palácio do Planalto. http://www.planalto.gov.br/ccivil_03/_Ato2007-2010/2009/Lei/L11947.htm

Brunori, G. (2007). Local food and alternative food networks: A communication perspective. Anthropology of food, S2. https://doi.org/10.4000/aof.430

Cauchick-Miguel, P. A., Fleury, A., Mello, C. H. P., Nakano, D. N., Lima, E. P. D., Turrioni, J. B., Ho, L. L., Morabito, R., Costa, S. E. G. D., Martins, R. A., Sousa, R., \& Pureza, V. (2018). Metodologia de Pesquisa em Engenharia de Produção e Gestão de Operações (3ºd). Elsevier.

Cervo, A. L., Bervian, P. A., \& Silva, R. da. (2009). Metodologia científica. Pearson Prentice Hall.

Coelho, F. C., Coelho, E. M., \& Egerer, M. (2018). Local food: Benefits and failings due to modern agriculture. Scientia Agricola, 75(1), 84-94. https://doi.org/10.1590/1678-992x-2015-0439

Darolt, M. R., Lamine, C., Brandenburg, A., Alencar, M. D. C. F., \& Abreu, L. S. (2016). Alternative Food Networks And New Producer-Consumer Relations in France And in Brazil. Ambiente \& Sociedade, 19(2), 1-22. https://doi.org/10.1590/1809-4422ASOC121132V1922016

Duarte, S. C. de L., \& Thomé, K. M. (2016). Short food supply chain: Estado da arte na academia brasileira. Estudos Sociedade e Agricultura, 23(2). https://revistaesa.com/ojs/index.php/esa/article/view/575

Eriksen, S. N. (2013). Defining local food: Constructing a new taxonomy - three domains of proximity. Acta Agriculturae Scandinavica, Section B - Soil \& Plant Science, 63(sup1), 47-55. https://doi.org/10.1080/09064710.2013.789123

Feenstra, G. (2002). Creating Space for Sustainable Food Systems: Lessons from the Field. Agriculture and Human Values, 19(2), 99-106. https://doi.org/10.1023/A:1016095421310

FNDE. (2020). Portal do FNDE Fundo Nacional de Desenvolvimento da Educação_PNAE. https://www.fnde.gov.br/index.php/programas/pnae

Fontefrancesco, M. F. (2018). The Slow Food Model: A Road for Small-Scale Productions in a Globalised Market. https://doi.org/10.22004/AG.ECON.292517

Galli, F., \& Brunori, G. (2013). Short Food Supply Chain as drivers of sustainable development. Laboratorio di studi rurali Sismondi,. http://www.foodlinkscommunity.net/fileadmin/documents_organicresearch/foodlinks/CoPs/evidence-document-sfsc-cop.pdf

Gaucher-Petitdemange, L. B. (2018). Short Food Supply Chain Initiatives and Their Potential For Sustainability In São Paulo State-Dissertação (MPGI)— Escola de Administração de Empresas de São Paulo. Fundação Getúlio Vargas.

Gil, A. Carlos. (2008). Como elaborar projetos de pesquisa (4ºd). Atlas.

Gong, T. (Charles), Battese, G. E., \& Villano, R. A. (2019). Family farms plus cooperatives in China: Technical efficiency in crop production. Journal of Asian Economics, 64, 101129. https://doi.org/10.1016/j.asieco.2019.07.002

Goodman, D. (2002). Rethinking Food Production-Consumption: Integrative Perspectives. Sociologia Ruralis, 42(4), 271-277. https://doi.org/10.1111/14679523.00216

Goodman, D. (2003). The quality 'turn' and alternative food practices: Reflections and agenda. Journal of Rural Studies, 19(1), 1-7. https://doi.org/10.1016/S0743-0167(02)00043-8

Google $\quad$ Maps. $\quad$ Mapa $\quad$ Satélite. map.gosur.com/pt/?gclid=Cj0KCQjwrsGCBhD1ARIsALILBYq7_OOqiKe_pFAkQTMTpKRHkvaFnc1XQUSaOZxX_c6dLHOuaEjFmgIaAk7bEALw_wcB Hinrichs, C. C. (2003). The practice and politics of food system localization. Journal of Rural Studies, 19(1), 33-45. https://doi.org/10.1016/S07430167(02)00040-2

IBGE, B. I. of G. and S.- 
Research, Society and Development, v. 10, n. 14, e273101419619, 2021

(CC BY 4.0) | ISSN 2525-3409 | DOI: http://dx.doi.org/10.33448/rsd-v10i14.19619

(2019). Agricultural Census 2017. IBGE. https://sidra.ibge.gov.br/pesquisa/censo-agropecuario/censo-agropecuario-2017.

IBGE, B. I. of G. and S.-. (2021). Inflation Index. https://www.ibge.gov.br/explica/inflacao.php

IBGE, I. B. de G. e E.-. (2020, novembro 27). Consumer Price Index —IPCA. Séries históricas. https://portalibre.fgv.br/

Mancini, M., Menozzi, D., Donati, M., Biasini, B., Veneziani, M., \& Arfini, F. (2019). Producers' and Consumers' Perception of the Sustainability of Short Food Supply Chains: The Case of Parmigiano Reggiano PDO. Sustainability, 11(3), 721. https://doi.org/10.3390/su11030721

Marsden, T., Banks, J., \& Bristow, G. (2000). Food Supply Chain Approaches: Exploring their Role in Rural Development. Sociologia Ruralis, 40(4), 424-438 https://doi.org/10.1111/1467-9523.00158

Martinez, S., Hand, M., Pra, M. D., Pollack, S., Ralston, K., Smith, T., Vogel, S., Clark, S., Lohr, L., Low, S., \& Newman, C. (2010). Local Food Systems Concepts, Impacts, and Issues. Economic Research Report, 97, 80.

Matte, A., Neske, M. Z., Borba, M. F. S., Waquil, P. D., \& Schneider, S. (2016). Mercado de cadeias curtas na pecuária familiar: Um processo de relocalização no território Alto Camaquã no Sul do Rio Grande do Sul/Brasil. Redes, 21(3), 137-158. https://doi.org/10.17058/redes.v21i3.5578

Ministério da Cidadania. (2020). Compras Governamentais. http://mds.gov.br/assuntos/seguranca-alimentar/programa-de-aquisicao-de-alimentos-paa/comprasgovernamentais

Pivoto, D., Mores, G. de V., Silva, R. F. da, \& Finocchio, C. P. S. (2016). Cadeias Curtas de Suprimentos de Alimentos: Uma Oportunidade para os Produtores Rurais?

https://www.researchgate.net/publication/304893058_Cadeias_curtas_de_suprimentos_de_alimentos_uma_oportunidade_para_os_produtores_rurais

Pozzebon, L., Rambo, A. G., \& Gazolla, M. (2017). As Cadeias Curtas das Feiras Coloniais e Agroecológicas: Autoconsumo e Segurança Alimentar e Nutricional. Desenvolvimento em Questão, 16(42), 405. https://doi.org/10.21527/2237-6453.2018.42.405-441

Renting, H., Marsden, T. K., \& Banks, J. (2003). Understanding Alternative Food Networks: Exploring the Role of Short Food Supply Chains in Rural Development. Environment and Planning A: Economy and Space, 35(3), 393-411. https://doi.org/10.1068/a3510

Sacchi, G., Cei, L., Stefani, G., Lombardi, G., Rocchi, B., Belletti, G., Padel, S., Sellars, A., Gagliardi, E., Nocella, G., Cardey, S., Mikkola, M., Ala-Karvia, U., Macken-Walsh, À., McIntyre, B., Hyland, J., Henchion, M., Bocci, R., Bussi, B., ... Vasvari, G. (2018). A Multi-Actor Literature Review on Alternative and Sustainable Food Systems for the Promotion of Cereal Biodiversity. Agriculture, 8(11), 173. https://doi.org/10.3390/agriculture8110173

Scarabelot, M., \& Schneider, S. (2012). As Cadeias Agroalimentares Curtas e Desenvolvimento Local - Um Estudo de Caso no Município de Nova Veneza/SC. Revista Faz Ciência, 14(19), 101.

Swaminathan, M. S., \& Kesavan, P. C. (2017). 2014 International Year of Family Farming: A boost to evergreen revolution. World Scientific. http://www.worldscientific.com/worldscibooks/10.1142/10279

Todorovic, V., Maslaric, M., Bojic, S., Jokic, M., Mircetic, D., \& Nikolicic, S. (2018). Solutions for More Sustainable Distribution in the Short Food Supply Chains. Sustainability, 10(10), 3481. https://doi.org/10.3390/su10103481

Tošović-Stevanović, A., Ćalović, D., Lalić, G., Žuža, M., \& Cvijanović, G. (2020). Comparative analysis of the economic potential of the small and family farms in the Republic of Serbia and Romania. Ekonomika Poljoprivrede, 67(3), 667-681. https://doi.org/10.5937/ekoPolj2003667T

Triches, R. M., \& Grisa, C. (2015). Entre mudanças e conservadorismos: Uma análise dos programas de aquisição de alimentos (PAA e PNAE) a partir da retórica da intransigência. Revista Nera, 18(26). https://revista.fct.unesp.br/index.php/nera/article/view/3569/2904

Vitterso, G., Torjusen, H., Laitala, K., Tocco, B., Biasini, B., Csillag, P., de Labarre, M. D., Lecoeur, J.-L., Maj, A., Majewski, E., Malak-Rawlikowska, A., Menozzi, D., Török, Á., \& Wavresky, P. (2019). Short Food Supply Chains and Their Contributions to Sustainability: Participants' Views and Perceptions from 12 European Cases. Sustainability, 11(17), 4800. https://doi.org/10.3390/su11174800

Wilkinson, J. (1999). Cadeias Produtivas para Agricultura Familiar. Organizações Rurais \& Agroindustriais. Revista de Administração da UFLA, l(1). http://revista.dae.ufla.br/index.php/ora/article/view/299 